\title{
Lexical choice of modal expressions
}

\author{
Ralf Klabunde \\ Department of Linguistics \\ Ruhr-Universität Bochum \\ Germany \\ klabunde@linguistics.rub.de
}

\begin{abstract}
This paper describes a model of the choice of modal verbs and modal particles. The choice mechanism does not require a modality-specific input as, e.g., a modal logical formula. Instead semantic (modal force) and pragmatic constraints (speech act marking) are applied to the available information on the whole and constrain the set of modal candidates to those that are appropriate in the respective contexts. The choice model is realized in the CAN system that generates recommendations about courses of study.
\end{abstract}

\section{Motivation}

Modality is a ubiquitous phenomenon. Speakers use modals very frequently to express that a state of affairs is not simply true or false, but that the propositional content of the respective sentence must be evaluated against some background information. For example, a sentence like she must study computer science expresses that, given some contextual information, it follows necessarily from this information that she studies computer science. Pragmatically, in the same context, the use of the modal verb must is able to signal the existence of a certain speech act, for example an advice.

Such an estimation of the validity of a proposition against contextual restrictions and signalling a speech act at the same time is rather the rule than the exception so that it is not surprising that modality concerns all relevant processing levels in NLG.

If we take the standard division of labour for an NLG system (cf. Reiter and Dale 2000), basically each module affects modality:
- Trivially, content selection is a modality-related task since a decision must be drawn which content shall be modalized.

- Discourse structuring may be modalitysensitive for two reasons: first, some rhetorical relations suggest the use of modals within their arguments, ${ }^{1}$ and second, if information is grouped, this chunk might serve as conversational background triggering the use of modals.

- Since sentence connectives can bear modal meanings (Blühdorn 2004), sentence aggregation is modalitysensitive as well.

- Modals are no content words, but they are polysemous lexical items and candidates for lexical choice.

- Even the generation of referring expressions is sensitive to modality because pronouns behave differently in certain modal contexts (a phenomenon labelled in formal semantics as modal subordination, see Roberts 1989).

- Finally, the grammatical realization requires modal-specific grammar rules.

Although modality is anything but a peripheral phenomenon, it did not receive much attention in research on NLG yet. This is even more astonishing if one considers generated texts produced by NLG systems. Many systems generate texts with modals, but the use of these modals is not driven by semantic decisions. For example, the STOP system (Reiter et al. 2003) generates letters which contain modal verbs

\footnotetext{
1 The examples given on the RST website (http://www.sfu.ca/rst/index.hmtl) show this very clearly.
} 
and/or conditionals (whose meaning is closely linked to modality) as, e.g., However, you might like to be a non-smoker if it was easy to stop. The question-answering system described in Moriceau (2006) uses web page extracts in addition to the generated answer. These extracts contain modal expressions (e.g., it could also be explained by random movements).

ADVISOR II (Elhadad 1995) is a generation system that covers exactly the same domain as the CAN system described in this paper. Its generated sentences contain modal verbs (e.g., You have little experience with writing papers.

So it could be difficult.), and it realizes several of the speech acts that play a role in CAN as well. However, since ADVISOR II is based on a theory of argumentation that focuses on scalars and lexical items with a scalar semantics, respectively, the choice of modals is not driven by the underlying theory. Lexical choice concerns primarily scalar adjectives, connectives, and judgement determiners.

In general, the papers on the mentioned systems suggest that the use of these modal expressions is not based on a meaningful representation of modality or processes that systematically result in the use of appropriate modal expressions. Rather these systems seem to realize modals by means of template-based approaches. A semantically or pragmatically motivated choice does not occur.

\subsection{Aim of this paper}

In this paper, we present such a model of the choice of modals. This model is integrated into the CAN system (conceptualization for modal expressions), an NLG system that generates recommendations for courses of study (Klabunde 2007). Currently, the choice of modals is confined to modal verbs like können (can) or müssen (must) and modal particles (ja, doch; these particles have no direct counterparts in English). The model does not require a content representation as formulas of some modal logic. The only concession to modality is the existence of more than one content plan. We operate with ordinary content plans created by a planner, and simulate the semantics of modal operators by means of quantification. Additionally, we formulate pragmatic conditions as speech act markers. The combination of semantic with pragmatically motivated conditions allows the selection of modal verbs and particles, respectively.

In what follows, we will first describe the semantic and pragmatic particularities of modal verbs and particles and some relevant analyses of these modals in formal semantics and pragmatics (section 2). Based on these approaches, we present the CAN system in section 3. The choice of modals in CAN is described in detail in section 4 . We conclude this paper with an outline of our future work.

\section{Modal verbs and particles}

Modality is a blurred concept that centers around the notions of possibility and necessity (cf. Kratzer 1981; Papafragou 2006, Werner 2006, and many others). Independent of what modal expression is used, a modal sentence expresses that the propositional content of the sentence possibly or necessarily holds with respect to some contextual restrictions.

\subsection{Modal verbs}

Standard approaches to the semantics of modal verbs emphasize the relevance of two parameters for a semantic analysis: the modal force, i.e. the expression of possibility or necessity, and contextual restrictions by means of a conversational background. Some German examples shall demonstrate the relevance of these parameters:

(1) Du musst den Semantik-Kurs besuchen You must attend the semantics course Modal force: necessity

No restriction on the conversational background

(2) Du sollst den Semantik-Kurs besuchen You are to attend the semantics course Modal force: necessity

Preferably a teleological, deontic or epistemic conversational background

(3) Du darfst den Semantik-Kurs besuchen You may attend the semantics course Modal force: possibility

Preferably a deontic conversational background

Meaning differences between modal verbs are explained by different admissible modal forces and conversational backgrounds. For example, 
the difference between müssen and sollen is just the class of admissible backgrounds: both express necessity as modal force, but while müssen is not confined to specific conversational backgrounds, sollen requires a teleological, a deontic, or an epistemic background.

In Kratzer's (1981) seminal work - inspired by modal logics - propositions and conversational backgrounds are represented as sets of possible worlds, so that the whole approach boils down to set-theoretic operations on possible world sets. For our approach it is especially important that the semantics of the modal operators (necessity) and $\diamond$ (possibility) is traced back to universal and existential quantification over possible worlds, respectively: if [p] denotes the set of possible worlds, where the proposition $p$ holds, and $\mathrm{R}(\mathrm{w})$ is the set of accessible possible worlds for a given world $w$, then: $[\square(\mathrm{p})]=\lambda \mathrm{w}$. $(\mathrm{R}(\mathrm{w}) \subseteq[\mathrm{p}])$, and $[\diamond(\mathrm{p})]=\lambda \mathrm{w}$. $(\mathrm{R}(\mathrm{w}) \cap[\mathrm{p}] \neq$ $\varnothing)$.

For example, the sentence you must attend the semantics course is true iff, given an actual world $\mathrm{w}$, all worlds that are accessible from $\mathrm{w}$ belong to the set of worlds in which the addressee attends the semantics course.

Although truth-conditions do not play an essential role for the choice of modals, modal forces do. We describe in section 4 how modal forces are modelled by quantification over plan nodes.

In addition to semantic constraints, modal verbs may also serve as speech act markers (cf. Zeevat 2003). Many modal verbs are associated with preferred conversational backgrounds, and this background suggests a certain speech act. For example, dürfen (may) expresses possibility as modal force and can be used if a deontic background becomes relevant. With such a background, dürfen expresses a permission.

\subsection{Modal particles}

Contrary to modal verbs, modal particles do not have a semantics at all; their function is to relate the propositional content to the speech situation. For example, $j a$ as modal particle indicates the speaker's evidence that the propositional content is true:

(4) Du besuchst ja den Semantik-Kurs

You are attending the semantic course - as we both know
Stressed doch marks a contradiction with the listener's assumptions or - with normal intonation - that the content is probably present in the common ground:

(5) Du besuchst DOCH/doch den SemantikKurs

You are attending the semantics course - I am right/ am I right?

The particles always express possibility as modal force, since the speaker signals that according to his belief state it is not definitely the case that the propositional content is true. Furthermore, the particles are typically used with an epistemic conversational background.

Zeevat (2004) points out that particles function as context- and speech act markers. As context markers, they signal the existence of a specific relation between the common ground $\mathrm{CG}$ and proposition $\phi$. For example, the particle $j a$ signals the relation $\operatorname{old}(\mathrm{CG}, \phi)$.

As for speech act marking, the modal particles behave analogous to modal verbs. The pragmatic function of the particles is to map the default speech act - the assertion - to a non-default one as, e.g., a reminder or a recommendation. If the particles function as speech act markers, their meaning may be represented as planning operators (cf. Appelt 1985 for an axiomatic approach to speech act planning). In this case, context marking belongs to the preconditions, and using the particle has certain effects with respect to the speaker's goal and the listener's information state.

In choosing modal particles we benefit from the vicinity of the formal characteristics of modal particles to planning operators. As described in section 4.2, we formulate speech acts as operator-like rules that manipulate the common ground.

\section{The CAN system}

The CAN system supports students in choosing courses for their course of study within the B.A. program at the University of Bochum. The users provide the system with the courses they attended so far, the maximal number of semesters they would like to study, and the courses they want to attend. Based on this information, the system generates recommendations/pieces of advice etc. which 
courses the user should/must/can/may take. The architecture of the system is given in figure 1 .

Content determination and linearization is realized by a forward planner. The planner produces a sequence of plans that are all of equal value. More than one plan is needed in order to have alternative scenarios as "possible worlds" at hand, which is a prerequisite for the use of any modal expression.

Since students have to major in two independent areas, plans can contain conflicts. For example, it could be the case that the user selects two courses that take place at the same time. In these cases the existence of the conflict will be linguistically indicated and the conflict must be resolved. Conflicts are resolved either by considering the internal hierarchy of the course types (obligatory courses are ranked higher than optional ones), or - if both courses are of equal value - by the user.

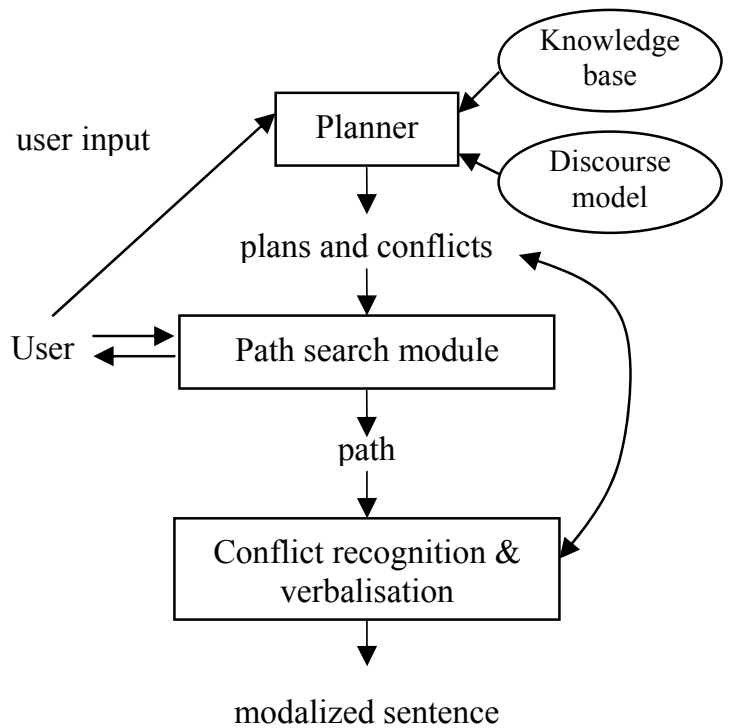

Fig. 1: Architecture of CAN

The modals are chosen by considering semantic and pragmatic constraints as described in section 4.

Currently, the system is able to choose the following modal expressions: müssen (must), können (can), wollen (want), dürfen (may), nicht brauchen (does not need to), the subjunctive sollten (should), and the particles $j a$ and doch.
The sentences are realized by means of sentential templates with slots for the courses, semesters, and modal expressions.

\section{The choice of modals in CAN}

The basic idea in the choice of modals is to use semantic and pragmatic constraints as filters for all modal candidates.

The conversational background fixes those modals that can be used at all. Based on this initial set, the computed modal force restricts the initial set to those modals that express that force. Finally, we check whether the speech acts associated with the remaining modals are appropriate in the respective context.

\subsection{Semantic constraints}

The conversational background is constituted by the application domain. Since the knowledge base contains information about the conditions of study, we are primarily dealing with deontic uses of the modals. The user's input as well as the information provided so far are stored in a discourse model that constitutes an epistemic background. $^{2}$

The modal force is computed by quantification over plan nodes. Basically, the content planner works in a STRIPS-like fashion (Fikes et al. 1972). In order to achieve a definition of modal forces in planning terms, it might be useful to remind some definitions:

A plan $\pi=\left\langle\alpha_{1}, \ldots, \alpha_{n}\right\rangle$ is a sequence of tasks $\alpha_{i}$. A plan graph $G$ is a set of plans which have the same starting node and the same final node: $\mathrm{G}=$ $\left\{\pi_{1}, \ldots, \pi_{\mathrm{m}}\right\}$ such that $\forall \pi_{\mathrm{i}}: \alpha_{1}, \alpha_{\mathrm{n}} \in \pi_{\mathrm{i}}$.

We use a simplified version of operator definitions that does without a delete list of items to be removed from the current state description: an operator is a pair $\langle\mathrm{P}, \mathrm{E}\rangle$ with $\mathrm{P}$ being a formula as precondition and $\mathrm{E}$ a set of formulas that describes the effects of the action. States are modified just by extending the current state by the effects of an instance of an operator: $\mathrm{M}_{\mathrm{i}}=\mathrm{M}_{\mathrm{i}-1} \cup$ E. A plan $\pi=\left\langle\alpha_{1}, \ldots, \alpha_{\mathrm{n}}\right\rangle$ will be

2 We should note that our approach is actually too simple to treat all epistemic uses. Epistemic interpretations express the speaker's estimation of the probability that a state of affairs comes true; we are just able to check whether some information was already given or is assumed to be new for the user. 
accepted if all tasks $\alpha_{i}$ are applicable in the corresponding states: $\mathrm{M}_{\mathrm{i}-1} \mid-\mathrm{P}_{\mathrm{\alpha i}}$

Given these standard definitions, the conditions for the modal forces are as follows:

$\square\left(\alpha_{j}\right)$ iff $\forall \pi_{i} \in G: M \alpha_{j-1} \mid-P_{\alpha j}$

$\diamond\left(\alpha_{\mathrm{j}}\right)$ iff $\exists \pi_{\mathrm{i}} \in \mathrm{G}: \mathrm{M} \alpha_{\mathrm{j}-1} \mid-\mathrm{P}_{\alpha \mathrm{j}}$

\subsection{Pragmatic constraints}

Our formulation of pragmatic constraints is based on Zeevat's $(2003,2004)$ work on speech act marking. Basically, Zeevat describes speech act markers (focus markers, modal particles, and others) as planning operators with preconditions and effects. Three speech acts and their constraints shall demonstrate how speech act marking works in our approach.

\subsubsection{Permissions}

Generally, a permission expresses that the addressee's goal is compatible with the goals of the speaker. Preconditions for realizing this speech act are:

- The speaker is socially superordinate to the listener. We believe that this essential condition is satisfied due to the user's acceptance of CAN as a supporting system.

- The propositional content of the sentence is compatible with the plan graph.

Context marking is not necessary. The system checks whether the user's favoured courses $\left\{c_{1}\right.$, $\left.\ldots, c_{n}\right\}$ can be mapped onto at least one of the generated plans: $\exists \pi_{\mathrm{i}}: \forall \mathrm{c}_{\mathrm{j}}: \mathrm{c}_{\mathrm{j}} \in \pi_{\mathrm{i}}$.

If these conditions are satisfied, the modal verb dürfen (may) is used since (1) it is an admissible modal verb for the underlying deontic background, (2) it expresses possibility as modal force, i.e. the mentioned compatibility of the conversational background with the propositional content (the user's courses), and due to the first condition mentioned above - it may be used as illocutionary indicator of permissions.

\subsubsection{Reminders}

Reminders draw on the discourse record as epistemic conversational background. The preconditions concern context marking: the content must already be suggested in the conversational background.

We are able to adopt Zeevat's (2004:102) characterisation who takes the relation old as context marker:

$\operatorname{old}(\mathrm{CG}, \phi)$ iff $\mathrm{CG} \mid=\operatorname{suggested}(\phi)$, and suggested is defined by means of a set of operators $\left\{\mathrm{O}_{1}, \ldots, \mathrm{O}_{\mathrm{n}}\right\}$ like $x$ dreams that, $x$ believes that etc.: suggested $(\phi) \leftrightarrow \phi \vee \mathrm{O}_{1}$ $\operatorname{suggested}(\phi) \vee \ldots \vee \mathrm{O}_{\mathrm{n}}$ suggested $(\phi)$.

Since we are not dealing with attitude contexts, our approach is a scaled-down version of Zeevat's proposal. The system checks whether the proposition had been inserted into the discourse record some time ago (in this case, CG $\mid=\phi$ is trivially satisfied). If this is the case, the modal particle $j a$ or doch is used because these particles expresses possibility as modal force (compatibility of the propositional content with a plan) and signal the reminder.

\subsubsection{Recommendations}

Recommendations declare a certain state of affairs as advantageous with respect to some goal. Computationally, this is an optimization problem: attending a course in a specific semester is advantageous for the completion of the course of study, if its attendance optimizes the number of semesters for that completion. If some course $c$ becomes relevant - either it was selected by the user or is given in the knowledge base - the precondition is: $\exists \pi_{\mathrm{i}}, \pi_{\mathrm{j}}$ with $\mathrm{c} \in \pi_{\mathrm{i}}, \pi_{\mathrm{j}}$ and $\left|\pi_{\mathrm{i}}\right|<\left|\pi_{\mathrm{j}}\right|$.

If such an optimal attendance exists, the subjunctive form sollte (should) is used. Sollte expresses possibility, may be used for deontic backgrounds, and is an illocutionary indicator of recommendations.

\subsection{Example outputs}

The following three examples show the contextdependent choice of modals, depending on the user's input, the information in the knowledge base, and the previous course of the interaction with the system. Note that the multiple occurrence of modals within one clause is possible:

- The user selects a course of type „synchronic linguistics“: 
CAN: Wenn Sie einen Kurs [Typ

Synchronie] im Grundkursmodul „Germanistische Linguistik“ besuchen wollen, dann müssen Sie den Grundkurs Germanistische Linguistik, Teil 2 besuchen.

If you want to attend a synchronic linguistics course in the basic module „Germanic Linguistics“, then you must attend part 2 of the basic course on Germanic Linguistics.

The generated conditional sentence contains a modal verb in the if-clause (wollen, want) and in the main clause (müssen, must). Wollen is used because the user declares attending a course of some type as his goal. This goal is compatible with at least one plan, viz. the plan that declares such a course in the current semester. Since this course must necessarily be attended to achieve the user's goal (every plan with the user's goal as final task contains this course), müssen is used. Speech act-related conditions are not relevant for this sentence.

- The user selects the semantics course again, after some other course selections:

CAN: Sie dürfen doch dieses Semester den Kurs "Semantik" nicht belegen, weil Sie nicht alle Voraussetzungen erfüllen.

You must not attend the semantics course, because you do not satisfy all prerequisites - did you forget this?

The generated sentence contains the negated modal verb dürfen (may) and the modal particle doch. The former (nicht dürfen) is used because there is no plan containing the wanted course in the respective semester (incompatibility of the user's input with the generated plans). For the same reason, the preconditions of a permission are not applicable. The particle has been chosen because the user selected the same course some time ago. The incompatibility is derived from the discourse model so that the sentence is a reminder on speech act level. The sentence also explains why the course is incompatible with each plan.

- The user selects two courses with overlapping hours:

CAN: Sie wollen „Strukturen einer Einzelsprache" und „Einführung in die
Linguistik" besuchen. Allerdings überschneiden sie sich zeitlich. Sie müssen eine andere Auswahl treffen.

You want to attend „Structures of a Language“ and „Introduction to Linguistics“. Both courses temporally overlap, however. You must make a different decision.

This example shows the linguistic output in case of a conflict. The modal verb wollen expresses the compatibility of the user's goal with a plan. This plan contains the conflict mentioned in the second sentence. The use of müssen in the final sentence is motivated by the necessity to find a conflict-free plan.

\section{Summary and future work}

The approach to the choice of modals described in this paper does not require modal logic formulas as content representations. However, since the use of modals signals talking about alternative scenarios/ worlds, one needs a way of representing these alternatives. We solved this problem by letting the planner create all possible plans for the same initial and final state. This bundle of equal plans allows us to determine the modal force by means of quantifying plan nodes. This semantic analysis restricts the set of modal candidates to those items that are able to express the determined modal force. A further diminution of this set is achieved by considering speech-act related conditions the modals must satisfy.

Our future work comprises broadening the set of modals (especially modal adverbs and syntactic constructions with modal meanings), a more elaborated treatment of modal forces, and the evaluation of the system's behaviour.

Thus far we treat modal forces from a traditional viewpoint: there are two forces and nothing in between. However, there are several linguistic means to signal different degrees of modal forces (various modal adverbs, the subjunctive, and others). Quantifying over the plan nodes should be a suitable means to model these varying modal forces. Just as generalized quantifiers express different relations between two sets, we should be able to trace back the modal forces to relations between all available nodes and those that express propositional units. 
Finally, we are planning to provide CAN with a web interface in order to gain comments on the linguistic adequacy of the generated modals. Comments from students who played around with the system seem to suggest that the modal verbs are appropriate but the use of the modal particles could be bemusing. The use of modal particles seems to be deeply rooted in ordinary, "real" conversation so that some students were confused when CAN generates a sentence with a chummy connotation. The evaluation shall clarify whether the modals support the acceptance of the expressed content.

\section{Acknowledgements}

I thank Alexander Linke and Arne Ruhnau for their implementation work in Perl and the reviewers for their helpful comments.

\section{References}

Appelt, D.E. (1985) Planning English sentences. Cambridge University Press, Cambridge.

Blühdorn, H. (2004) Temporalkonnektoren: Einleitung. In H. Blühdorn, E. Breindl and U. Hermann (eds.), Brücken schlagen. Grundlagen der Konnektorensemantik, de Gruyter, Berlin/New York, pp. 125-136.

Elhadad, M. (1995) Using argumentation in text generation. Journal of Pragmatics, 24, pp. 189-220.

Fikes, R., P. Hart \& N.J. Nilsson (1972) Learning and executing generalized robot plans. Artificial Intelligence, 3(4), pp. 251-288.

Klabunde, R. (2007) Generating modals. Proceedings of the Seventh International Workshop on Computational Semantics, Tilburg; pp. 342-345.

Kratzer, A. (1981) The notional category of modality. In H.-J. Eikmeyer \& H. Rieser (eds.) Words, Worlds, and Contexts. De Gruyter, Berlin, pp. 38-74.

Moriceau, V. (2006) Generating intelligent numerical answers in a question-answering system. Proceedings of the Fourth International Natural Language Generation Conference, Sydney, pp. 96103.

Papafragou, A. (2006) Epistemic modality and truth conditions. Lingua, 116, pp. 1688-1702.

Reiter, E. and Dale, R. (2000) Building Natural Language Generation Systems. Cambridge. Cambridge University Press.

Reiter, E., R. Robertson \& L. Osman (2003) Lessons from a Failure: Generating Tailored Smoking
Cessation Letters. Artificial Intelligence, 144, pp. 41-58.

Roberts, C. (1989) Modal subordination and pronominal anaphora in discourse. Linguistics \& Philosophy, 12 (6), pp. 683-721.

Werner, T. (2006) Future and non-future modal sentences. Natural Language Semantics, 14, pp. 235-255.

Zeevat, H. (2003) The syntax semantic interface of speech act markers. Proceedings of Diabruck 03. 7th Workshop on the Semantics and Pragmatics of Dialogue.

Zeevat, H. (2004) Particles: Presupposition Triggers, Context Markers or Speech Act Markers. In R. Blutner \& H. Zeevat (eds.) Optimality Theory and Pragmatics. Palgrave Macmillan, London, pp. 91111. 